\title{
Shells with Thickness Distension
}

\author{
A. DiCarlo* $\quad$ P. Podio-Guidugli ${ }^{\dagger} \quad$ W. O. Williams ${ }^{\ddagger}$
}

July 23, 1999

to appear in a special issue on Multi-Field Theories

of the International Journal of Solids and Structures

\section{Contents}

1 Introduction $\quad 2$

2 Three-Dimensional Cauchy Continua 4

3 Shells: Geometry $\quad 7$

3.1 Body Structure . . . . . . . . . . . . . . . . . 7

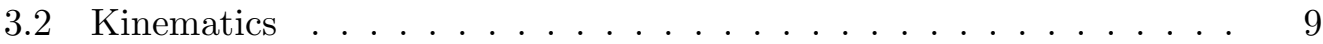

4 Shells: Balance Laws $\quad 11$

4.1 Balance of Base Forces . . . . . . . . . . . . . . . . . . . . . . . 11

4.2 Balance of Torques . . . . . . . . . . . . . . . . . . 12

4.3 Balance of Director Forces . . . . . . . . . . . . . . . . . 13

4.4 Summary of Balance Laws . . . . . . . . . . . . . . . . . . . . 14

5 Shells: Basic Constitutive Issues $\quad \mathbf{1 5}$

5.1 Inertial Forces and Couples . . . . . . . . . . . . . . . . . 16

5.2 Symmetry Condition on Generalized Stresses . . . . . . . . . . . . 18

5.3 Reactive Stress Fields and Null Stress Fields . . . . . . . . . . . . . 19

\footnotetext{
*Dipartimento di Scienze dell'Ingegneria Civile, Università degli Studi "Roma Tre", Via Corrado Segre 60, I-00146 Roma, Italy (adc@uniroma3.it)

${ }^{\dagger}$ Dipartimento di Ingegneria Civile, Università di Roma "Tor Vergata", Via di Tor Vergata 110, I-00133 Roma, Italy (ppg@uniroma2.it)

${ }^{\ddagger}$ Department of Mathematical Sciences, Carnegie Mellon University, Schenley Park, Pittsburgh, PA 15213-3890 USA (wow@cmu.edu)
} 
6 Reflections on Deductive Approaches to Shell Balances 22

6.1 Rigid Motions . . . . . . . . . . . . . . . . . 22

6.2 Deduction by Invariance . . . . . . . . . . . . . . . . . . . 22

6.3 Deduction by Equipollence and Equipower . . . . . . . . . . . . . 23

7 Application to Linear Thickness Oscillations 25

7.1 Free Vibrations of Linearly Elastic Plates . . . . . . . . . . . . . . . 25

7.2 Shearing and Thickness Oscillations . . . . . . . . . . . . . . 27

7.3 Propagation of Thickness-Distention Waves . . . . . . . . . . . 29

7.4 Enriched Kinematics . . . . . . . . . . . . . . . . . . . . . . . . 31

\begin{abstract}
A deductive approach to shell theory is presented, within which a shell is regarded as a constrained three-dimensional continuum with special body structure: more precisely, admissible deformations are given a restricted form, presumed to be consistent with the special shape and partitionability of a shelllike body.

In addition to the standard balance equations of forces and torques, an extra balance equation is derived, allowing for a description of dilatation or contraction in the thickness dimension. As an illustrative application, the free oscillations of linearly elastic plates - in particular, thickness-distension wavesare studied.
\end{abstract}

\title{
1 Introduction
}

Plate and shell theories are two-dimensional continuum models, endowed with various degrees of structure. The points of view taken in constructing the balance equations and constitutive equations of such theories can be grouped into three categories.

First, one may take a direct approach, that introduces the shell as a twodimensional structured continuum: kinematic descriptors are prescribed, balance equations are obtained from first principles, and constitutive prescriptions are posited $a b$ initio.

The other two approaches both deduce the balance equations and constitutive assumptions for plates and shells from those of a parent three-dimensional theory (and hence we call them deductive). In one method, the dependent theory is found as the limit of an approximation in which one dimension (the thickness) of the body is made to tend to zero. The other method, which we use in this paper, regards plates and shells as three-dimensional bodies with special shape and partitionability, whose deformations are restricted to be, in some precise sense, "shell-like" (Sections 2 and 3$)$. 
A moot point in this third approach is that the balance equations of the twodimensional theory can be derived from those of the parent theory in various, not entirely equivalent ways. We choose here a procedure (Section 4) which seems to us economical, in that it borrows concepts from the direct method (DiCarlo, forthcoming), and satisfactorily general, because it works properly for any class of deformations selected and because it provides in a uniform way all of the relevant balance equations. Some other deductive methods are discussed in Section 6 .

The implementation of the constitutive theory - of which we here treat only some basic preliminaries common to all material classes (Section 5) - is straightforward, as the pertinent constitutive information is obtained from that embodied in the parent theory, either by standard methods of the theory of constrained continua (PodioGuidugli, 1989; Lembo and Podio-Guidugli, 1991; Nardinocchi and Podio-Guidugli, 1994; Podio-Guidugli, forthcoming) or by some "mixed" variant of them that uses complementary assumptions on deformation and stress rather than on deformation alone (Teresi and Tiero, 1997).

The main advantage of our approach is that it allows one to avoid a separate development of constitutive prescriptions, a seemingly inevitable step in the first approach ${ }^{1}$. Another advantage is that it offers an "intuitive" interpretation of the generalized forces and stresses arising in the theory ${ }^{2}$. In addition, the resulting theory need not be seen as an approximation, as is inherent in the second approach; accordingly, questions of degree of precision are bypassed, as is the difficulty of establishing that the approximate theory is a true homogenization of the parent theory.

The price paid for these advantages is that since the deformation class is special and hence the theory constrained, the posing of boundary-value problems is made more subtle by the presence of reactions to the constraints. We characterize the reactions by a partwise integral condition of null working; since we assign to three-dimensional shell-like bodies a peculiarly restricted partitionability, such a characterization is not equivalent to the usual pointwise algebraic condition. Here,

\footnotetext{
${ }^{1}$ This is a mixed blessing, however: provided the constitutive ingredients are properly tuned, the same direct theory can model the behavior not only of a thin, shell-like standard body, but also of something quite different - a latticed shell, for instance.

${ }^{2}$ In truth, such an interpretation is intuitive mostly for those already accustomed to the standard notion of stress, that is, virtually all students of continuum mechanics after Cauchy. Nonetheless, some notion of "generalized stresses" foreran what was to become the standard concept of stress; when introducing the latter, Cauchy himself wrote (1827): "The geometers who have investigated the equations of equilibrium or motion of thin plates or of surfaces . . have distinguished two kinds of forces, the one produced by dilatation or contraction, the other by the bending of these surfaces ... It has seemed to me that these two kinds of forces could be reduced to a single one, which ought to be called always tension or pression, a force which acts upon each element of a section chosen at will, not only in a flexible surface but also in a solid ...".
} 
we touch only briefly on the issue of choosing the three-dimensional reactive stress field that best supplements a two-dimensional shell solution. We point out, however, that an application of the second approach should help to clarify how the constraints introduced in the third approach weaken - and the associated reactive fields enfeeble - as the thickness goes to zero.

We choose the simplest class of admissible deformations that allows us to derive, beside the standard balance equations of forces and torques, a "through-thethickness" balance equation, which rules dilatation and contraction in this dimension. The kinematical and dynamical elements of the direct shell theory that correspond to ours are listed in the opening of Section 5; such a direct theory has the format of a continuum theory of two-dimensional bodies with microstructure (Capriz, 1989).

Our theory handles large deformations as well as small. To illustrate the sort of phenomena that can be accounted for, in our last section we give an application to linear elastodynamics of plates, studying in particular the propagation of thicknessdistension waves.

\section{Three-Dimensional Cauchy Continua}

In this section we establish the format which we will use to frame our shell theory, by applying it first to a three-dimensional Cauchy continuum. This format is a version, fully developed by DiCarlo (1996), of the classical method of virtual power (Germain, 1972; Germain, 1973a; Germain, 1973b; Maugin, 1980; Antman and Osborne, 1979). The notions and notations we use are conventional, with a few, carefully defined, exceptions.

We identify a body with its reference shape $\Omega$ (an open region in a threedimensional Euclidean manifold $\mathcal{E}$ ). Associated to $\Omega$ are (i) a family $\mathcal{M}$ of motions $f: \bar{\Omega} \times \mathbb{R} \rightarrow \mathcal{E}$, with each $f(\cdot, t)$ a deformation of $\Omega$, and (ii) a family $\mathbb{V}$ of test velocities $v: \bar{\Omega} \rightarrow \mathcal{V}$, with $\mathcal{V}$ the (oriented) translation space of $\mathcal{E}$. We assume that, at each fixed time $t \in \mathbb{R}, \mathbb{V}$ includes all realizable velocities

$$
v(x)=\dot{f}(x, t),
$$

where $f \in \mathcal{M}$ and a dot denotes time differentiation, and also all superposed rigid velocities

$$
v(x)=v_{\mathrm{o}}+\omega \times\left(f(x, t)-y_{\mathrm{o}}\right),
$$

with $v_{\mathrm{o}}, \omega \in \mathcal{V}, f \in \mathcal{M}, y_{\mathrm{o}} \in \mathcal{E}$. We also suppose $\mathbb{V}$ rich enough in smooth fields with arbitrarily small support that the standard localization arguments apply at any given point in $\Omega$ or its boundary $\partial \Omega$. 
Along with each motion we assign a (force, stress) pair

$$
((d, s), S) \text {. }
$$

At time $t, d(x, t)$, the distance force per unit volume (including the inertial force), is defined for $x \in \Omega, s(x, t)$, the applied traction per unit area, is defined for $x \in \partial \Omega$, and $S(x, t)$, the Piola stress, is defined for $x \in \bar{\Omega}=\Omega \cup \partial \Omega$.

Given a motion and a (force, stress) pair, we introduce two functionals, defined on velocities $v \in \mathbb{V}$ :

the force working

$$
\mathcal{F}(v)=\int_{\Omega} d \cdot v+\int_{\partial \Omega} s \cdot v
$$

and the stress working

$$
\mathcal{S}(v)=\int_{\Omega} S \cdot \operatorname{Grad} v
$$

and we call surfeit working (or simply working) their difference ${ }^{3}$ :

$$
\mathcal{W}(v)=\int_{\Omega} d \cdot v+\int_{\partial \Omega} s \cdot v-\int_{\Omega} S \cdot \operatorname{Grad} v .
$$

The principle of null working is the requirement that the surfeit working be zero over all test velocities:

$$
\mathcal{W}(v)=\mathcal{F}(v)-\mathcal{S}(v)=0, \quad v \in \mathbb{V} .
$$

We insert the identity

$$
S \cdot \operatorname{Grad} v=\operatorname{Div}\left(S^{\top} v\right)-v \cdot \operatorname{Div} S
$$

in (2.4b) and localize (2.5), to deduce that the principle of null working is equivalent to balance of forces, in the form

$$
\begin{array}{rlrl}
\operatorname{Div} S+d=0 & & \text { on } \Omega, \\
S \nu & =s & & \text { on } \partial \Omega,
\end{array}
$$

$\nu$ being the outer unit normal to $\partial \Omega$.

\footnotetext{
${ }^{3}$ Our force working corresponds to what is called outer working by DiCarlo (1996), while our stress working is the negative of the inner working in DiCarlo (1996). We envisage the working as a difference (whence the name surfeit working), since we prefer to interpret it as the virtual energy dissipation. To be precise, we should require the working functional to be continuous on $\mathbb{V}$, after assigning a topology to the space of test velocities. We do not find it necessary to elaborate upon this point here, and we content ourselves with the cavalier treatment of such issues which is standard in continuum mechanics.
} 
We supplement the principle of null working with two basic constitutive requirements, each applied to the stress working over a body-part:

$$
\mathcal{S}_{\Pi}(v)=\int_{\Pi} S \cdot \operatorname{Grad} v,
$$

a body-part $\Pi$ being a body-like subset of $\Omega$.

The first requirement is that the stress working over each part should vanish for each superposed rigid test-velocity field $(2.2)^{4}$. The gradient of such a velocity takes the form

$$
\operatorname{Grad} v=W F \text {, }
$$

where $F=\operatorname{Grad} f(\cdot, t)$ is the current deformation gradient, and where $W=\omega \times$ is a constant skew tensor field. Since $W$ may be chosen arbitrarily, and since we suppose that each point of $\bar{\Omega}$ is contained in parts of arbitrarily small size, we arrive at the standard result

$$
\operatorname{skw}\left(S F^{\top}\right)=0 .
$$

The second requirement is that the stress should be prescribed by constitutive equations only to the extent that it performs work on realizable velocities (2.1). This is to apply to each part, and is best stated by saying that the stress may have an arbitrary additive term, the reactive stress $S_{R}$, which obeys

$$
\int_{\Pi} S_{R} \cdot \operatorname{Grad} \dot{f}=0
$$

for each part $\Pi$ and each motion $f \in \mathcal{M}$ (Antman and Marlow, 1991; Antman, 1995; Podio-Guidugli, 1995). Arbitrariness of parts implies that, at each point of $\bar{\Omega}$,

$$
S_{R} \cdot \dot{F}=0, \quad \dot{F}=\operatorname{Grad} \dot{f}, \quad f \in \mathcal{M} .
$$

\section{Remark}

- When we specialize to a theory of small deformations from a stress-free placement, the force balance (2.7) retains its form; this is not so for the symmetry condition (2.10), which becomes

$$
\text { skw } S=0 \text {. }
$$

\footnotetext{
${ }^{4}$ Principles of invariance - of the force working under change of observer or of the energy balance under superposition of rigid motions - were advanced formally by Noll (1963) and Green and Rivlin (1964), respectively; these authors regarded all of their invariance postulates as equivalent to balance principles (of mass, force, and torque). The point of view we adopt here, that the invariance of stress working under superposed rigid velocities should be regarded as a constitutive requirement, was asserted by Germain (1972). We elaborate on this matter in the last remark of Section 5.2.
} 
Likewise, the reaction characterization (2.11b), consistent with (2.12), becomes

$$
S_{R} \cdot \dot{E}=0, \quad \dot{E}=\operatorname{sym}(\operatorname{Grad} \dot{f}), \quad f \in \mathcal{M} .
$$

(here $E$ is the linear strain related to the motion $f$; see Section 7.1).

\section{Shells: Geometry}

Our point of view is that of Podio-Guidugli (1995), Podio-Guidugli (forthcoming): plates and shells should be regarded as Cauchy continua with a special body structure and a special kinematics consistent with that structure.

\subsection{Body Structure}

We call a Cauchy body shell-like if it has a reference shape which is a right cylinder:

$$
\Omega=\{p+\zeta e \mid p \in \mathfrak{P}, \zeta \in \mathfrak{I}\},
$$

with $\mathfrak{P}$, the base surface, a flat two-dimensional region of $\mathcal{E}$, $e$ a unit vector normal to $\mathfrak{P}$, and $\mathfrak{I}=(-\varepsilon,+\varepsilon)$ an interval. (We use $\varepsilon$ to suggest that the thickness of $\Omega$ is small: e.g., length $(\mathfrak{I})<<\operatorname{diam}(\mathfrak{P})$.) In the following, we identify points in $\Omega$ with pairs in the Cartesian product $\mathfrak{P} \times \mathfrak{I}$ :

$$
p+\zeta e \simeq(p, \zeta)
$$

The collection of body parts for $\Omega$ consists only in shell-like parts $\mathfrak{Q} \times \mathfrak{I}$, with $\mathfrak{Q}$ a part of $\mathfrak{P}$; the crux of this specialization is that the atoms of the body structure are the fibers $\{p\} \times \mathfrak{I}$, with $p \in \mathfrak{P}$. The boundary of $\Omega$ divides naturally into the lateral boundary $\partial \mathfrak{P} \times \mathfrak{I}$ and the upper and lower faces $\mathfrak{P} \times\{+\varepsilon\}, \mathfrak{P} \times\{-\varepsilon\}$. We use the notation

$$
\phi^{ \pm}(p)=\phi(p, \pm \varepsilon)
$$

for the restrictions of a field $\phi$ to the faces.

Given an integrable field $\phi$ on $\Omega$, we consider its integrals only over subregions that are shell-like parts of $\Omega$, so that

$$
\int_{\mathfrak{Q} \times \mathfrak{I}} \phi=\int_{\mathfrak{Q}}\left(\int_{\mathfrak{I}} \phi(\cdot, \zeta) d \zeta\right)
$$

and

$$
\int_{\partial(\mathfrak{Q} \times \mathfrak{I})} \phi=\int_{\mathfrak{Q}}\left(\phi^{+}+\phi^{-}\right)+\int_{\partial \mathfrak{Q}}\left(\int_{\mathfrak{I}} \phi(\cdot, \zeta) d \zeta\right)
$$


If $\varphi$ is a real-valued function on $\Omega$, we set

$$
\operatorname{Grad} \varphi=\operatorname{grad} \varphi+\varphi^{\prime} e,
$$

where $\operatorname{grad} \varphi$ is the orthogonal projection of $\operatorname{Grad} \varphi$ onto $\mathfrak{P}(\operatorname{grad} \varphi=P(\operatorname{Grad} \varphi)$, with $P=1-e \otimes e, 1$ being the identity on $\mathcal{V}$ ), and where $\varphi^{\prime}$ is the derivative of $\varphi$ along the normal direction $\left(\varphi^{\prime}=(\operatorname{Grad} \varphi) \cdot e\right)$. Likewise, for $u$ a $\mathcal{V}$-valued mapping on $\Omega$,

$$
\begin{aligned}
\operatorname{Grad} u & =\operatorname{grad} u+u^{\prime} \otimes e,{ }^{5} \\
\operatorname{Div} u & =\operatorname{tr}(\operatorname{Grad} u)=\operatorname{tr}(\operatorname{grad} u)+u^{\prime} \cdot e=\operatorname{div} u+u^{\prime} \cdot e,
\end{aligned}
$$

where $\operatorname{grad} u=(\operatorname{Grad} u) P$; accordingly, for $H$ a $(\mathcal{V} \otimes \mathcal{V})$-valued mapping on $\Omega$,

$$
\operatorname{Div} H=\operatorname{div} H+H^{\prime} e \text {. }
$$

In $(3.5 \mathrm{~b})$ a prime denotes, as in $(3.5 \mathrm{a})$, differentiation along the normal to $\mathfrak{P}$. The following identities deserve notice:

$$
\operatorname{div} u=\operatorname{div}(P u), \quad \operatorname{div} H=\operatorname{div}(H P) .
$$

\section{Remarks}

- Even within a treatment that allows for arbitrarily large deformations, the requirement that the reference shape be cylindrical may seem to impose drastic limits on the scope of our theory. Actually, an assumption such as (3.1) places no serious restrictions: if weakened as to hold only partwise (that is, for each part of a collection covering $\Omega$ ), it would not rule out such cases as toroidal shells. In applications, one may find it convenient to use a reference shape which is not locally flat; however, there is no reason to embark here on the differential-geometric complications related to a non-uniform unit normal field over $\mathfrak{P}$; such complications add nothing to the generality of the theory.

- Were one to develop a theory of rod-like bodies, one would still consider reference shapes with the representation (3.1), this time typically with $\operatorname{diam}(\mathfrak{P})<<\operatorname{length}(\mathfrak{I})$, body parts of the form $\mathfrak{P} \times(\alpha, \beta)$, with $(\alpha, \beta) \subset \mathfrak{I}$, and cross-sections $\mathfrak{P} \times\{\zeta\}$, with $\zeta \in \mathfrak{I}$, as atoms of the resulting body-structure.

\footnotetext{
${ }^{5}$ We define $(a \otimes b) u=(b \cdot u) a, u \in \mathcal{V}$.
} 


\subsection{Kinematics}

We introduce restricted classes of motions and test velocities, both consistent with the above body structure.

First, without any loss of generality, we decompose the motion of a point $(p, \zeta)$ of $\bar{\Omega}$ into the motion of the associated point $p$ of the base surface $\mathfrak{P}$ plus the motion relative to $p$ :

$$
f(p, \zeta, t)=g(p, t)+\mathfrak{g}(p, \zeta, t)
$$

where

$$
g(p, t)=f(p, 0, t), \quad \mathfrak{g}(p, \zeta, t)=f(p, \zeta, t)-f(p, 0, t) .
$$

We call $g(\cdot, t)$ the base deformation. Our fundamental assumption is that the relative motion leaves each fiber straight and that it is linear on each fiber:

$$
\mathfrak{g}(p, \zeta, t)=\zeta l(p, t), \quad l(p, t)=\alpha(p, t) Q(p, t) e .
$$

Here the scalar $\alpha$, which must be positive, measures the fiber stretch, that is, the thickness dilatation or contraction of the shell. The tensor $Q$ is orthogonal, so that $|Q e|=1$, and the difference between $Q e$ and the unit normal to the image $g(\mathfrak{P}, t)$ of the base surface measures the transverse shear of the shell. Motivated by experience with the direct theories mentioned in the Introduction, we call the vector $l$ the director. Combining (3.6a) and (3.7) we obtain the general form of the restricted class of motions we shall consider:

$$
f(p, \zeta, t)=g(p, t)+\zeta \alpha(p, t) Q(p, t) e,
$$

a form parametrized by $g, \alpha$, and $Q .{ }^{6}$

We note that (3.8) leads to deformation gradients of the form

$$
F=\operatorname{grad} g+l \otimes e+\zeta \operatorname{grad} l .
$$

Likewise, at any fixed time $t \in \mathbb{R}$, motions of the form (3.8) generate realizable velocities of the form

$$
\begin{aligned}
v(p, \zeta) & =\dot{g}(p, t)+\zeta(\dot{\alpha}(p, t) Q(p, t)+\alpha(p, t) \dot{Q}(p, t)) e \\
& =\dot{g}(p, t)+\zeta(\psi(p, t) 1+\Psi(p, t)) l(p, t) .
\end{aligned}
$$

Here we have introduced the fiber stretching $\psi$ and the director spin $\Psi$ :

$$
\psi=\dot{\alpha} \alpha^{-1}, \quad \Psi=\dot{Q} Q^{\top} .
$$

\footnotetext{
${ }^{6}$ Such a parametrization, while convenient, is redundant: for all orthogonal $R$ such that $R e=e$, $Q R$ and $Q$ correspond to the same motion; hence, only two degrees of freedom are associated with the parameter $Q$. In Section 4.2 we shall see that this has substantial consequences.
} 
Consistent with (3.10), we utilize the following family of test velocities:

$$
v(p, \zeta)=w(p)+\zeta(\lambda(p) 1+\Lambda(p)) l(p, t),
$$

with the vector field $w$, the scalar field $\lambda$ and the skew tensor field $\Lambda$ appropriately smooth, but otherwise arbitrary in both value and support. The latter condition ensures that we can apply standard localization arguments at any given point in $\overline{\mathfrak{P}}=\mathfrak{P} \cup \partial \mathfrak{P}$. At any time $t$, the subspace of $\mathbb{V}$ spanned by (3.11) includes all rigid velocities (2.2) superposed on deformations of the form (3.8) (cf. Section 6.1).

\section{Remarks}

- We do not require the director field $l$ to be normal to the current image of the base surface, as is the case in the standard theories of plates and shells such as the Kirchhoff-Love theory; theories of the Reissner-Mindlin type do account for transverse shear, but not for thickness distention.

- A more general form of (3.7) could be considered, namely,

$$
\mathfrak{g}(p, \zeta, t)=\gamma(p, \zeta) l(p, t),
$$

with $\gamma(p, \cdot)$ a prescribed nonlinear function on $\mathfrak{I}$. For example, given a $\zeta$-dependent mass density $\rho$, one could then choose $\gamma$ to ensure that the corresponding densitymoment vanishes:

$$
\int_{\mathfrak{I}} \gamma(p, \zeta) \rho(p, \zeta) d \zeta=0
$$

a condition that simplifies the treatment of generalized inertial forces ( $c f$. Section 5.1). However, it is neither difficult nor interesting to modify the formulae below to treat the case in which $\gamma(p, \cdot)$ is not the identity on $\mathfrak{I}$.

- Conditions of compatibility, both local and global, are associated to the restricted class of motions we consider. We do not discuss these conditions here, since they place no restriction on the specification of test velocities.

- We have chosen the form (3.8) for the admissible motions because it is the simplest one which allows for the effects we wish to model, but of course there are other kinematic formulations which are consistent with the shell-like body structure of Section 3.1. The representation (3.7) could be generalized to

$$
\mathfrak{g}(p, \zeta, t)=\mathfrak{h}(\pi(p, t), \zeta), \quad \mathfrak{h}(\pi(p, t), 0)=0,
$$

where $\pi$ takes values in a manifold $\mathcal{P}$ of parameters. At any fixed $t$, the realizable velocities would have the form

$$
v(p, \zeta)=\dot{g}(p, t)+\mathfrak{H}_{(\pi(p, t), \zeta)} \dot{\pi}(p, t),
$$


with $\mathfrak{H}_{(\pi(p, t), \zeta)}$ the differential of $\mathfrak{h}(\cdot, \zeta)$ at $\pi(p, t)$; test velocities at time $t$ would then have the form

$$
v(p, \zeta)=w(p)+\mathfrak{H}_{(\pi(p, t), \zeta)} \varpi(p),
$$

with $\varpi(p) \in T_{\pi(p, t)} \mathcal{P}$, the tangent space to $\mathcal{P}$ at $\pi(p, t)$; appropriate generalized stresses could then be constructed, just as we do below in the special case (3.8), where $\mathcal{P}=\mathcal{V}, \pi(p, t)=l(p, t)$, and $\mathfrak{h}(\pi(p, t), \zeta)=\zeta l(p, t)$. The only caution in building such models is that the class of motions engendered by (3.14) should be closed under composition with rigid motions, and hence invariant under change of observer ( $c f$. Section 6.1).

\section{Shells: Balance Laws}

We proceed now to apply the principle of null working to shell-like bodies for the restricted collection of test velocities (3.11). Since the working functional is linear, we choose to examine one by one the cases of variation of the free parameters $w$, $\Lambda$, and $\lambda$.

\subsection{Balance of Base Forces}

First, we set $\lambda=0, \Lambda=0$, and consider test velocities of the form

$$
v(p, \zeta)=w(p)
$$

with gradients

$$
\operatorname{Grad} v=\operatorname{grad} w .
$$

It follows that the force working (2.4a) takes the form

$$
\mathcal{F}(w)=\int_{\mathfrak{P}} b \cdot w+\int_{\partial \mathfrak{P}} n \cdot w,
$$

where the base bulk-force $b$ and the base boundary-traction $n$ are defined to be, respectively,

$$
\begin{aligned}
b(p, t) & =\int_{\mathfrak{I}} d(p, \zeta, t) d \zeta+s^{+}(p, t)+s^{-}(p, t), \quad p \in \mathfrak{P}, \\
n(p, t) & =\int_{\mathfrak{I}} s(p, \zeta, t) d \zeta, \quad p \in \partial \mathfrak{P} .
\end{aligned}
$$

The stress working becomes

$$
\mathcal{S}(w)=\int_{\mathfrak{P}} N \cdot \operatorname{grad} w
$$


where the mean stress $N$ is defined to be

$$
N(p, t)=\int_{\mathfrak{I}} S(p, \zeta, t) d \zeta, \quad p \in \overline{\mathfrak{P}} .
$$

Notice that only the base stress $N P$, that is, the mean stress $N$ times the projection $P=1-e \otimes e$, contributes to the working (4.5), and hence to the balance of base forces. The reason for introducing and naming $N$ itself will surface in the next subsection.

In the present context, the principle of null working is the assertion that

$$
\mathcal{W}(w)=\mathcal{F}(w)-\mathcal{S}(w)=0,
$$

for all test velocities $w$. Using the base-surface analogue of identity (2.6), namely,

$$
N \cdot \operatorname{grad} w=\operatorname{div}\left(N^{\top} w\right)-w \cdot \operatorname{div} N,
$$

we obtain, after localization of (4.7), the balance equations for the base forces:

$$
\begin{aligned}
\operatorname{div} N+b=0 & \text { on } \mathfrak{P} \\
N \nu=n & \text { on } \partial \mathfrak{P},
\end{aligned}
$$

where $\nu$ is the outer unit normal to $\partial \mathfrak{P}$ tangent to $\mathfrak{P} .{ }^{7}$

\subsection{Balance of Torques}

Next, for each given motion and for each fixed time $t$, we consider test velocities of the form

$$
v(p, \zeta)=\zeta \Lambda(p) l(p, t)
$$

with gradients

$$
\operatorname{Grad} v=(\Lambda l) \otimes e+\zeta \operatorname{grad}(\Lambda l) .
$$

With (4.10) the force working becomes

$$
\mathcal{F}(\Lambda)=\int_{\mathfrak{P}}(c \otimes l) \cdot \Lambda+\int_{\partial \mathfrak{P}}(m \otimes l) \cdot \Lambda,
$$

where the bulk couple $c$ and the boundary couple $m$ are defined to be, respectively,

$$
\begin{aligned}
c(p, t) & =\int_{\mathfrak{I}} \zeta d(p, \zeta, t) d \zeta+\varepsilon\left(s^{+}(p, t)-s^{-}(p, t)\right), \quad p \in \mathfrak{P}, \\
m(p, t) & =\int_{\mathfrak{I}} \zeta s(p, \zeta, t) d \zeta, \quad p \in \partial \mathfrak{P} .
\end{aligned}
$$

\footnotetext{
${ }^{7}$ Hence $\nu=P \nu ;$ moreover, $\operatorname{div} N=\operatorname{div}(N P) \quad($ recall $(3.5 \mathrm{e}))$.
} 
Further, with (4.11), the stress working takes the form

$$
\mathcal{S}(\Lambda)=\int_{\mathfrak{R}}((q \otimes l) \cdot \Lambda+M \cdot \operatorname{grad}(\Lambda l)),
$$

where the mean transverse-traction $q$ and the couple stress $M$ are defined to be, respectively,

$$
\begin{aligned}
q(p, t) & =\int_{\mathfrak{I}} S(p, \zeta, t) e d \zeta=N(p, t) e \\
M(p, t) & =\int_{\mathfrak{I}} \zeta S(p, \zeta, t) d \zeta,
\end{aligned}
$$

for $p$ in $\overline{\mathfrak{P}}$; definition (4.6) has been used to relate the mean transverse-traction $q$ to the mean stress $N$. An application of the identity (4.8), with $N$ replaced by $M$ and $w$ by $\Lambda l$, followed by use of the divergence theorem, yields the following expression for $\mathcal{S}(\Lambda)$ :

$$
\mathcal{S}(\Lambda)=\int_{\mathfrak{P}}((q-\operatorname{div} M) \otimes l) \cdot \Lambda+\int_{\partial \mathfrak{P}}((M \nu) \otimes l) \cdot \Lambda
$$

It follows that the principle of null working localizes to the balance equations for torques:

$$
\begin{aligned}
(\operatorname{div} M-N e+c) \times l=0 & \text { on } \mathfrak{P} \\
(M \nu) \times l=m \times l & \text { on } \partial \mathfrak{P} .
\end{aligned}
$$

Note that, as anticipated in footnote 6 on page 9 , each of the vectorial equations (4.17) has two scalar equivalents, since the test velocities (4.10) compose a twoparameter collection at each point.

\subsection{Balance of Director Forces}

Finally, for each given motion and for each fixed time $t$, we select the test velocities

$$
v(p, \zeta)=\zeta \lambda(p) l(p, t)
$$

whose gradients are

$$
\operatorname{Grad} v=\lambda l \otimes e+\zeta \operatorname{grad}(\lambda l) .
$$

On replacing $\Lambda l$ by $\lambda l$ in (4.12), the force working becomes

$$
\mathcal{F}(\lambda)=\int_{\mathfrak{P}}(c \cdot l) \lambda+\int_{\partial \mathfrak{P}}(m \cdot l) \lambda,
$$


with the bulk couple $c$ and the surface couple $m$ given by the definitions (4.13). We call the constructs $c \cdot l$ and $m \cdot l$ the director bulk-force and the director boundary-force. Further, with (4.19), (4.15), and (4.8), the stress working takes the form

$$
\begin{aligned}
\mathcal{S}(\lambda) & =\int_{\mathfrak{P}}((q \cdot l) \lambda+M \cdot \operatorname{grad}(\lambda l)) \\
& =\int_{\mathfrak{P}}((q-\operatorname{div} M) \cdot l) \lambda+\int_{\partial \mathfrak{P}}((M \nu) \cdot l) \lambda .
\end{aligned}
$$

As a consequence of (4.20) and (4.21), the principle of null working localizes to the balance equations for the director forces:

$$
\begin{aligned}
(\operatorname{div} M-N e+c) \cdot l=0 & \text { on } \mathfrak{P} \\
(M \nu) \cdot l=m \cdot l & \text { on } \partial \mathfrak{P} .
\end{aligned}
$$

These equations, which are absent in the standard theories of plates and shells of Kirchhoff-Love and Reissner-Mindlin, allow for a description of thickness distention.

\subsection{Summary of Balance Laws}

The balance laws we posit for shells are, at each point $p$ of the base surface $\mathfrak{P}$, the balance of base forces (4.9a), that is:

$$
\operatorname{div} N+b=0
$$

and the balance of couples (encompassing both balance of torques (4.17a) and balance of director forces $(4.22 \mathrm{a})$ )

$$
\operatorname{div} M-N e+c=0 .
$$

When the contributions lumped into the base bulk-force $b$ and the bulk couple $c$ (in particular, inertial forces and couples) are specified, and constitutive relations are stipulated for the mean stress $N$ and for the couple stress $M$ in terms of the history of motion, one obtains a system of evolution equations ( $c f$. Section 5.1). This system has to be supplemented by appropriate initial conditions and by boundary conditions (perhaps only on part of $\partial \mathfrak{P})$ of the form indicated by $(4.9 \mathrm{~b}),(4.17 \mathrm{~b})$ and $(4.22 \mathrm{~b})$, that is:

$$
\begin{aligned}
& N \nu=n, \\
& M \nu=m .
\end{aligned}
$$




\section{Remarks}

- Notice that only the base couple-stress $M P$ contributes to the workings (4.14) and (4.21), and hence to the balance of couples (recall (3.5e)); $M e$, the piece of information complementary to $M P$, does not enter a shell theory like ours, which is based on the simple kinematics (3.8); however, $M e$ surfaces as soon as a kinematically richer theory is considered ( $c f$. Section 7.4). Likewise, while both the base stress $N P$ and the mean transverse-traction $N e$ play a role in our present theory, only the base stress would appear in a poorer theory where (3.8) is replaced by

$$
f(p, \zeta, t)=g(p, t),
$$

and the only balance is (4.9).

- While we have called constructions like $c$ and $m$ couples (see (4.13)), the true couples are their tensor products with the director $l$, namely $c \otimes l$ and $m \otimes l$. The skew parts of such products are faithfully represented by the cross products $c \times l, m \times l$ (which we have called torques), while the working expended by their symmetric parts is characterized by the traces $c \cdot l, m \cdot l$ (called director forces).

- Equation (4.23b) and the companion boundary equation (4.24b) (each of which is equivalent to three independent scalar equations) can also be obtained directly by testing the working functional on velocities of the form

$$
v(p, \zeta)=\zeta \varpi(p),
$$

parameterized by the director velocity $\varpi$, an arbitrary vector field (recall (3.16)).

\section{Shells: Basic Constitutive Issues}

Our shell theory is based on the following working-conjugate lists of dynamic and kinematic descriptors:

$$
\mathbf{d}=(N P, N e, M P), \quad \mathbf{k}=(\operatorname{grad} g, l, \operatorname{grad} l),
$$

with the pairing $\int_{\mathfrak{P}} \mathbf{d} \cdot \dot{\mathbf{k}}$ giving the stress working expended along a motion of type (3.8) (cf. (5.12), (3.11), (3.10)). Of this two-dimensional structure, the pair $(N P, \operatorname{grad} g)$ corresponds to the pair $(S, \operatorname{Grad} f)$ typical of a three-dimensional continuous body of Cauchy type; the remaining two conjugate pairs in $(\mathbf{d}, \mathbf{k})$, namely, $(N e, l)$ and $(M P, \operatorname{grad} l)$ depict the dynamical and kinematical structure additional to Cauchy's (Capriz, 1989).

Within a direct construction of shell theory based on these descriptors, one would study constitutive mappings delivering $\mathbf{d}$ in terms of the history of $\mathbf{k}$. Although we need not take up these matters, since we work within a theory induced 
from a three-dimensional theory, we do consider three constitutive issues that are preliminary and common to any choice of a shell material-class: we characterize the two-dimensional bulk interactions of inertial nature, with a view toward formulating evolution problems; we restrict contact interactions by a symmetry condition in terms of $\mathbf{d}$ and $\mathbf{k}$ that corresponds to the classical three-dimensional condition of symmetry for the Cauchy stress; and we discuss the reactive stress fields that maintain the kinematical constraint implicit in our definition of admissible motions.

\subsection{Inertial Forces and Couples}

Part of the distance force $d$ in (2.3) arises from inertia. We write

$$
d=d^{i n}+d^{n i},
$$

with $d^{i n}$ the inertial force, and $d^{n i}$ the non-inertial force per unit volume. Granted that along a motion $f$

$$
d^{i n}(x, t)=-\rho(x) \ddot{f}(x, t),
$$

with the mass density $\rho$ a prescribed positive scalar field on $\Omega$, and taking into account the fact that we have confined attention to motions of the form:

$$
f(p, \zeta, t)=g(p, t)+\zeta l(p, t)
$$

( $\left.c f .(3.6 \mathrm{a}),(3.7)_{1}\right)$, it is not difficult to derive the corresponding generalized inertial forces for shells. Differentiating (5.4) twice with respect to time, we have

$$
\ddot{f}(p, \zeta, t)=\ddot{g}(p, t)+\zeta \ddot{l}(p, t) .
$$

To find the inertial contribution to the base bulk-force (4.4a) we compute

$$
b^{i n}(p, t)=\int_{\mathfrak{I}} d^{i n}(p, \zeta, t) d \zeta=-(\mu(p) \ddot{g}(p, t)+I(p) \ddot{l}(p, t)),
$$

where we have introduced the mean density

$$
\mu(p)=\int_{\mathfrak{I}} \rho(p, \zeta) d \zeta
$$

and the first density-moment

$$
I(p)=\int_{\mathfrak{I}} \zeta \rho(p, \zeta) d \zeta
$$

Similarly, we find that the inertial contribution to the bulk couple (4.13a) is

$$
c^{i n}(p, t)=\int_{\mathfrak{I}} \zeta d^{i n}(p, \zeta, t) d \zeta=-(I(p) \ddot{g}(p, t)+K(p) \ddot{l}(p, t)),
$$


where

$$
K(p)=\int_{\mathfrak{I}} \zeta^{2} \rho(p, \zeta) d \zeta
$$

is the second density-moment. Recalling the balance laws (4.23), and splitting the generalized bulk forces in accordance with (5.2):

$$
b=b^{i n}+b^{n i}, \quad c=c^{i n}+c^{n i},
$$

we arrive at the evolution equations:

$$
\begin{aligned}
\operatorname{div} N+b^{n i} & =\mu \ddot{g}+I \ddot{l}, \\
\operatorname{div} M-N e+c^{n i} & =I \ddot{g}+K \ddot{l} .
\end{aligned}
$$

Director time-derivatives may be expressed in terms of the fiber stretching $\psi$, the director spin $\Psi$, and their time derivatives. From $(3.7)_{2}$ and (3.10b) one gets

$$
\begin{aligned}
& i=(\psi 1+\Psi) l \\
& \ddot{l}=\left(\left(\dot{\psi}+\psi^{2}\right) 1+2 \psi \Psi+\dot{\Psi}+\Psi^{2}\right) l .
\end{aligned}
$$

To split (5.8b) into two equations, corresponding respectively to the balance of torques (4.17a) and of director forces (4.22a), we compute the cross and dot products of $\ddot{l}$ and $l$ :

$$
\begin{aligned}
\ddot{l} \times l & =-\alpha^{2}((\dot{\varsigma}+2 \psi \varsigma) \kappa+\varsigma \dot{\kappa}), \\
\ddot{l} \cdot l & =\alpha^{2}\left(\dot{\psi}+\psi^{2}-\varsigma^{2}\right),
\end{aligned}
$$

with $\alpha$ the fiber stretch, and the director spin $\Psi$ represented as

$$
\Psi=\varsigma \kappa \times,
$$

where $\varsigma$ is a scalar and $\kappa$ a unit vector perpendicular to $l$.

Finally, we record the formula for the kinetic-energy density on the base surface: along any motion (5.4) and for any surface-part $\mathfrak{Q}$,

$$
\begin{aligned}
\int_{\mathfrak{Q} \times \mathfrak{I}} \frac{1}{2} \rho|\dot{f}|^{2} & =\int_{\mathfrak{Q}} \frac{1}{2}\left(\mu|\dot{g}|^{2}+2 I \dot{g} \cdot \dot{l}+K|\dot{l}|^{2}\right) \\
& =\int_{\mathfrak{Q}} \frac{1}{2}\left(\mu|\dot{g}|^{2}+2 I \dot{g} \cdot \dot{l}+K \alpha^{2}\left(\psi^{2}+\varsigma^{2}\right)\right) .
\end{aligned}
$$

\section{Remarks}

- Note that, while $\mu$ and $K$ are necessarily positive, $I$ may well be zero; if it is zero, the balance of base forces and the balance of couples (5.8) are inertially uncoupled. 
- The inertial terms engendered by (5.10a) in the balance of torques and by (5.10b) in the balance of director forces both include the effects of fiber stretching and director spin, and hence couple the two equations. However, since the coupling terms are quadratic, they do not appear in a linear approximation.

\subsection{Symmetry Condition on Generalized Stresses}

Summing up the partial results (4.5), (4.14) and (4.21), we find the general representation of the stress working over a shell-like body-part $\mathfrak{Q} \times \mathfrak{I}$ as an integral over the surface-part $\mathfrak{Q}$ :

$$
\mathcal{S}_{\mathfrak{Q} \times \mathfrak{I}}(w, \varpi)=\int_{\mathfrak{Q}}(N \cdot(\operatorname{grad} w+\varpi \otimes e)+M \cdot \operatorname{grad} \varpi) .
$$

Here the velocity parameters $\lambda$ and $\Lambda$ together determine the director velocity

$$
\varpi(p)=(\lambda(p) 1+\Lambda(p)) l(p, t) .
$$

We now require the stress working over each shell-like part to vanish for each rigid test velocity superposed upon the current deformation, represented by

$$
\begin{aligned}
& w(p)=v_{\mathrm{o}}+\omega \times\left(g(p, t)-y_{\mathrm{o}}\right), \\
& \varpi(p)=\omega \times l(p, t)
\end{aligned}
$$

(cf. (2.2), (3.11)). This constitutive requirement parallels the one leading to (2.10) in the three-dimensional theory. The symmetry condition

$$
\operatorname{skw}\left(N(\operatorname{grad} g)^{\top}+(N e) \otimes l+M(\operatorname{grad} l)^{\top}\right)=0
$$

ensues, for all motions in the family (3.8). In the same way that (2.10) restricts the constitutive prescriptions of the stress $S,(5.14)$ restricts the constitutive prescriptions of the mean stress $N$ and the couple stress $M$.

\section{Remarks}

- Relation (5.14) can also be arrived at by inserting (3.9) into (2.10):

$$
\operatorname{skw}\left(S(\operatorname{grad} g)^{\top}+(S e) \otimes l+\zeta S(\operatorname{grad} l)^{\top}\right)=0,
$$

and then integrating (5.15) over fibers, with the use of definitions (4.6) and (4.15b) of $N$ and $M$. Thus, admissible constitutive prescriptions for $S$ engender admissible prescriptions for $N$ and $M$. 
- In a theory of small deformations from a stress-free placement, both $N$ and $M$ turn out to be symmetric; hence the linearization of (5.14),

$$
\operatorname{skw} N=0,
$$

is satisfied by fiat.

- In the case of general three-dimensional continua, following the principles set forth by Noll (1963) or Green and Rivlin (1964), one would consider the partwise working, i.e., the following functional on $\mathbb{V}$ :

$$
\begin{aligned}
\mathcal{F}_{\Pi}(v) & =\int_{\Pi} d \cdot v+\int_{\partial \Pi \cap \Omega}\left(S \nu_{\Pi}\right) \cdot v+\int_{\partial \Pi \cap \partial \Omega} s \cdot v \\
& =\int_{\Pi} d \cdot v+\int_{\partial \Pi}\left(S \nu_{\Pi}\right) \cdot v+\int_{\partial \Pi \cap \partial \Omega}(s-S \nu) \cdot v \\
& =\int_{\Pi}((\operatorname{Div} S+d) \cdot v+S \cdot \operatorname{Grad} v)+\int_{\partial \Pi \cap \partial \Omega}(s-S \nu) \cdot v,
\end{aligned}
$$

where $\Pi$ is a general part of $\Omega,((d, s), S)$ the (force, stress) pair introduced in (2.3), $\nu$ the outer unit normal to $\partial \Omega$, and $\nu_{\Pi}$ the outer unit normal to $\partial \Pi$. One would then obtain both the force balance (2.7) and the symmetry condition (2.10), by requiring that, for any part of $\Omega$, the partwise working (5.17) vanishes over any rigid test velocity (2.2) superposed on the current deformation. However, (2.10) would be thought of as a balance equation for torques, sharing a common status with (2.7).

In order to construct a two-dimensional shell theory in the same spirit, one would require the partwise working (5.17) to vanish only for any shell-like part $\Pi=\mathfrak{Q} \times \mathfrak{I}$ of $\Omega$, over any rigid test velocity superposed on the deformations given by (3.8). So doing, one would arrive at the balance equations for the base forces (4.9), plus the bulk balance equation for torques

$$
\operatorname{skw}\left(N(\operatorname{grad} g)^{\top}+(\operatorname{div} M+c) \otimes l+M(\operatorname{grad} l)^{\top}\right)=0 \quad \text { on } \mathfrak{P},
$$

supplemented by the boundary balance equation for torques (4.17b). Equation (5.18) should be contrasted with equation (5.14); both would have to be postulated in order to obtain our bulk balance equation for torques (4.17a).

\subsection{Reactive Stress Fields and Null Stress Fields}

Return for a moment to the characterization of the reactive stress in a threedimensional Cauchy body $\Omega$. Whenever the collections of body parts and realizable velocities are sufficiently rich, the pointwise characterization (2.11b) holds, implying 
that the reactive stress $S_{R}$ is everywhere null in $\bar{\Omega}$. For a shell-like three-dimensional body $\Omega=\mathfrak{P} \times \mathfrak{I}$, whose parts have the form $\mathfrak{Q} \times \mathfrak{I}$ and whose admissible motions have the form (3.8), the partwise characterization (2.11a) is appropriate. Since the parts $\mathfrak{Q}$ of $\mathfrak{P}$ can be chosen arbitrarily, (2.11a) implies that the reactive descriptor

$$
\begin{gathered}
\mathbf{d}_{R}=\left(N_{R} P, N_{R} e, M_{R} P\right), \\
N_{R}=\int_{\mathfrak{I}} S_{R} d \zeta, \quad M_{R}=\int_{\mathfrak{I}} \zeta S_{R} d \zeta,
\end{gathered}
$$

is everywhere null in $\overline{\mathfrak{P}}$, i.e.,

$$
\int_{\mathfrak{I}} S_{R} d \zeta=0, \quad\left(\int_{\mathfrak{I}} \zeta S_{R} d \zeta\right) P=0 \quad \text { on } \overline{\mathfrak{P}}
$$

Interestingly, a reactive stress field $S_{R}$ satisfying (5.20) may aid in better approximating the three-dimensional stress field $S$ in a shell-like three-dimensional body $\Omega$ : having solved the corresponding shell problem in $\mathfrak{P}$, the shell solution can be utilized to construct, with the use of the constitutive prescriptions, an "active" stress field $\widetilde{S}$ in $\bar{\Omega}$, and the arbitrariness left by conditions (5.20) in the choice of $S_{R}$ can be exploited to minimize the distance between the fields $S$ and $\left(\widetilde{S}+S_{R}\right)$.

More generally, in order to approximate $S$, the stress fields in $\bar{\Omega}$ associated to any null stress descriptor in $\mathfrak{P}$ can be used. For a three-dimensional Cauchy continuum a null stress ${ }^{0} S$ is any field that satisfies

$$
\begin{aligned}
\operatorname{Div}^{0} S=0 & \text { on } \Omega, \\
{ }^{0} S \nu=0 & \text { on } \partial \Omega
\end{aligned}
$$

(cf. (2.7)), a strong form of the equation

$$
\int_{\Omega}{ }^{0} S \cdot \operatorname{Grad} v=0 \quad \text { for all test fields } v .^{8}
$$

Likewise, a null stress descriptor ${ }^{0} \mathbf{d}=\left({ }^{0} N P,{ }^{0} N e,{ }^{0} M P\right)$ in a two-dimensional shell continuum satisfies

$$
\begin{aligned}
\operatorname{div}{ }^{0} N=0, \quad \operatorname{div}{ }^{0} M-{ }^{0} N e=0 & \text { on } \mathfrak{P}, \\
{ }^{0} N \nu=0, \quad{ }^{0} M \nu=0 & \text { on } \partial \mathfrak{P} ;
\end{aligned}
$$

\footnotetext{
${ }^{8}$ Condition (5.22) shows that null stresses perform no work on test velocities, while reactive stresses perform no work on the smaller collection of realizable velocities. $C f$. Truesdell and Toupin (1960, p. 547) and Gurtin (1972, p. 55) for discussions of slightly different notions of null stress.
} 
the associated stress fields in $\bar{\Omega}$, that we continue to indicate by ${ }^{0} S$ although they need not satisfy (5.21), are such that

$$
\int_{\mathfrak{I}}{ }^{0} S d \zeta={ }^{0} N, \quad\left(\int_{\mathfrak{I}} \zeta^{0} S d \zeta\right) P={ }^{0} M P
$$

Null stress fields are irrelevant to the development of the standard three-dimensional theory, and so are null generalized stresses to the development of a direct shell theory. Whether one chooses to introduce stress as a fundamental quantity in a working principle, or to construct it from force balances, null fields are unavoidable artifacts that can, however, be disposed of by simply ignoring them. But in a shell theory deduced from a parent three-dimensional theory according to an approach like ours, the stress fields obeying (5.23) and (5.24) can be used to approximate the three-dimensional stress field $S$. Precisely, if the motion of a three-dimensional shell-like body $\Omega$ is approximately described by the solution of the corresponding shell problem in $\mathfrak{P}$, this shell solution can be used to construct a constitutively determined, approximate stress field $\widetilde{S}$ in $\bar{\Omega}$; then a better approximation for $S$ can be sought by choosing among the fields ${ }^{0} S$ obeying (5.23), (5.24), and, in addition, satisfying the pointwise conditions:

$$
\begin{aligned}
\operatorname{Div}{ }^{0} S=-(\operatorname{Div} \widetilde{S}+d) & \text { on } \Omega, \\
{ }^{0} S^{+} e=-\left(\widetilde{S}^{+} e-s^{+}\right) & \text {on } \mathfrak{P} \times\{+\varepsilon\}, \\
{ }^{0} S^{-} e=-\left(\widetilde{S}^{-} e+s^{-}\right) & \text {on } \mathfrak{P} \times\{-\varepsilon\}, \\
{ }^{0} S \nu=-(\widetilde{S} \nu-s) & \text { on } \partial \mathfrak{P} \times \mathfrak{I} .
\end{aligned}
$$

We plan to elaborate on this idea, which has traits in common with a proposal by Antman (1995), in DiCarlo et al. (forthcoming).

\section{Remark}

- A scant supply of exact solutions to equilibrium problems for linearly elastic, three-dimensional plate-like bodies is available in the literature (see, e.g., Levinson (1985), Pan (1991), Rogers et al. (1992)). Conceivably, these solutions can serve as benchmarks to test and compare the approximations offered by one or another plate theory. In addition, for the three-dimensional stress field $\widetilde{S}$ induced by a given plate theory, these benchmark equilibria could help to assess whether a corrective field of the reactive and/or null types $S_{R}$ and ${ }^{0} S$ is in some sense "optimal". 


\section{Reflections on Deductive Approaches to Shell Bal- ances}

As we have pointed out in the Introduction, there are various ways to deduce the balance laws for shells from the parent three-dimensional theory. In this section we examine some of the alternatives, after a preliminary discussion of rigid motions.

\subsection{Rigid Motions}

The motion class we consider, although restricted, is closed under composition with rigid motions $r: \mathcal{E} \times \mathbb{R} \rightarrow \mathcal{E}$,

$$
r(y, t)=r_{\mathrm{o}}(t)+R(t)\left(y-y_{\mathrm{o}}\right),
$$

where $y_{\mathrm{o}}, r_{\mathrm{o}}(t) \in \mathcal{E}$, and $R(t)$ is orthogonal. In fact, if a motion $f$ is of the form

$$
f(p, \zeta, t)=g(p, t)+\zeta \alpha(p, t) Q(p, t) e,
$$

then the compound motion $\widehat{f}(\cdot, t)=r(\cdot, t) \circ f(\cdot, t)$ also belongs to the same class, since

$$
\widehat{f}(p, \zeta, t)=\widehat{g}(p, t)+\zeta \alpha(p, t) \widehat{Q}(p, t) e,
$$

with

$$
\begin{aligned}
\widehat{g}(p, t) & =r_{\mathrm{o}}(t)+R(t)\left(g(p, t)-y_{\mathrm{o}}\right), \\
\widehat{Q}(p, t) & =R(t) Q(t) .
\end{aligned}
$$

Correspondingly, all superposed rigid velocities (2.2) belong to the family of test velocities (3.11), being represented by (5.13); in particular, (5.13b) implies

$$
\begin{aligned}
\lambda(p) & =0, \\
\Lambda(p) & =\omega \times .
\end{aligned}
$$

\subsection{Deduction by Invariance}

Relation (6.3a) clearly shows that it would be impossible to derive our balance equations (4.22) for director forces from an invariance principle modelled after Noll's or Green and Rivlin's principles (Noll, 1963; Green and Rivlin, 1964), because such a principle would test the working solely on velocities which do not stretch the fibers ${ }^{9}$. There is, however, a method of deduction by invariance that yields both our balance

\footnotetext{
${ }^{9}$ Naghdi (1981, Subsection 3.2) states this frankly. After a direct postulation of conservation laws for shells, of mass (1), linear momentum (2), director momentum (3) and moment of momentum (4), he writes: "The conservation laws (1), (2) and (4) are equivalent to, and can be derived from
} 
of base forces (4.23a), (4.24a) and our balance of couples (4.23b), (4.24b) - as well as an infinite hierarchy of balances of "hypercouples". Naghdi (1972, Subsection $12 \delta$ ) - following Green et al. (1968) and Green and Naghdi (1970, 1971)-proposes to integrate over the thickness the local form of the energy balance, with the weights $\zeta^{k}(k=0,1,2, \ldots)$; and to require that each of the resulting "energy moments" be separately invariant under superposition of rigid motions.

A method as ad hoc and formal as Naghdi's, equivalent to it but simpler, consists in the weighted thickness integration of the three-dimensional balance equations (2.7). One integrates $\zeta^{k}$ times (2.7) and, since for any $k>0$

$$
\int_{\mathfrak{I}} \zeta^{k} \operatorname{Div} S d \zeta=\operatorname{div}\left(\int_{\mathfrak{I}} \zeta^{k} S d \zeta\right)-k\left(\int_{\mathfrak{I}} \zeta^{k-1} S d \zeta\right) e+\varepsilon^{k} S^{+} e-(-\varepsilon)^{k} S^{-} e,
$$

without recourse to invariance assumptions, one obtains the balances $(4.23),(4.24)$ for $k=0,1$ (using (4.6), (4.15)), along with denumerably many more balances. All of these would also follow from the principle of null working (2.5) for test velocities of the form

$$
v(p, \zeta)=\sum_{k=0}^{\mathcal{K}} \zeta^{k} \varpi_{k}(p),
$$

with $\mathcal{K}$ arbitrarily large and each $\varpi_{k}$ an arbitrary vector field (cf. (4.26)).

\subsection{Deduction by Equipollence and Equipower}

This subsection is based on lines of reasoning presented in Podio-Guidugli (forthcoming), where more details can be found.

For a three-dimensional Cauchy body $\Omega$, the balances of force and torque in the manner of Noll may be written in the form

$$
\int_{\Pi} d+\int_{\partial \Pi} S \nu_{\Pi}=0
$$

the conservation of energy (5) and the invariance conditions under superposed rigid body motions. The conservation law (3) for the director momentum must be postulated separately.". He had already voiced the same point of view in his Handbuch article (Naghdi, 1972), in the part devoted to the direct approach. In a footnote closing Subsection $8 \delta$, Naghdi writes: "The conservation law for the director momentum (3) cannot be deduced from the balance of energy and the invariance requirements under superposed rigid body motions. In their derivation of the field equations via the energy balance and the invariance requirements, Green et al. (1965) did not explicitly state a separate postulate for the integral form of the director momentum principle but assumed a local form of the equations of motion for the director couple which can be deduced from (3).". And later on (last paragraph of Subsection $9 \beta$ ): "In the context of an oriented surface, the equilibrium equations given by Ericksen and Truesdell (1958) are incomplete or restrictive; and this is mainly because their basic principles do not include a director momentum principle corresponding to (3)." 


$$
\int_{\Pi}\left(f-y_{\mathrm{o}}\right) \times d+\int_{\partial \Pi}\left(f-y_{\mathrm{o}}\right) \times\left(S \nu_{\Pi}\right)=0,
$$

for $\Pi$ an arbitrary part of $\Omega(c f$. (5.17)). For a shell-like body $\Omega=\mathfrak{P} \times \mathfrak{I}$ with parts $\Pi=\mathfrak{Q} \times \mathfrak{I}$, relations (6.6) take the form

$$
\begin{gathered}
\int_{\mathfrak{Q}} b+\int_{\partial \mathfrak{Q}} N \nu_{\mathfrak{Q}}=0, \\
\int_{\mathfrak{Q}}\left(\left(g-y_{\mathrm{o}}\right) \times b+\mathfrak{t}\right)+\int_{\partial \mathfrak{Q}}\left(\left(g-y_{\mathfrak{o}}\right) \times\left(N \nu_{\mathfrak{Q}}\right)+\mathfrak{T} \nu_{\mathfrak{Q}}\right)=0 .
\end{gathered}
$$

Here we have used (3.6a), (4.4a), (4.6) and, letting $\mathfrak{G}(p, \zeta, t)=\mathfrak{g}(p, \zeta, t) \times$, we have defined

$$
\begin{gathered}
\mathfrak{T}(p, t)=\int_{\mathfrak{I}} \mathfrak{G}(p, \zeta, t) S(p, \zeta, t) d \zeta, \quad p \in \overline{\mathfrak{P}} \\
\mathfrak{t}(p, t)=\int_{\mathfrak{I}} \mathfrak{g}(p, \zeta, t) \times d(p, \zeta, t) d \zeta+\mathfrak{g}^{+}(p, t) \times s^{+}(p, t)-\mathfrak{g}^{-}(p, t) \times s^{-}(p, t), p \in \mathfrak{P} .
\end{gathered}
$$

At each point $p$ in the closure of $\mathfrak{P}$, and for each unit vector $\nu$ perperdicular to $e$, the generalized contact action per unit base-length $(N \nu, \mathfrak{T} \nu)$ is equipollent (i.e., equal in force and torque) to the distribution over the fiber $\{p\} \times \mathfrak{I}$ of the contact force per unit area $S \nu$; likewise, the generalized bulk action per unit base-area $(b, \mathfrak{t})$ is equipollent to the distribution over the fiber of the distance force per unit volume $d$ and of the end tractions per unit area $s^{ \pm}$.

Having in this way deduced a two-dimensional dynamic structure (and the associated balances), one must still obtain a conjugate kinematic structure. In this connection, it must be noted that construction by equipollence yields generalized actions determined only to within a nilpollent system of actions. The ambiguity can be resolved, and a conjugate kinematics constructed, by use of the equipower principle: one requires that, for all motions in a given class, the working of the generalized actions $(b, \mathfrak{t}, N \nu, \mathfrak{T} \nu)$, possibly augmented by other nilpollent actions, equals the working of the Cauchy actions $(d, S \nu)$.

The larger the class of motions admitted, the less effective the equipollence principle in determining the generalized actions: as a key example, for the class (3.8) which we have considered, it fails to detect the director forces and their balance (DeSimone and Podio-Guidugli, 1995); indeed, the most general shell theory derived solely by equipollence is the Reissner-Mindlin theory, which obtains by restricting the class (3.8) by the additional requirement

$$
\alpha(p, t)=|l(p, t)|=1
$$

of fiber inextensibility. 


\section{Application to Linear Thickness Oscillations}

To illustrate the range of phenomena which can be modelled within our theory, we apply it to study the vibrations of linearly elastic plates.

In the first three subsections we obtain from (5.8) the basic evolution equations for plates of arbitrary response symmetry; we specialize these evolution equations to shearing and thickness oscillations of transversely isotropic plates; and we study the propagation in such plates of certain thickness-distension waves.

The key assumption of this paper is that the transverse fibers remain straight, and their stretch constant through the thickness, in any motion relative to the base surface $(c f .(3.7))$. In the last subsection we relax this assumption so as to allow for affine fiber stretch and constant, nonnull fiber curvature; our intention is to indicate how the governing equations both increase in number and change in structure, in order to handle such an enriched kinematics.

\subsection{Free Vibrations of Linearly Elastic Plates}

We begin by deriving the evolution equations for a linearly elastic plate of thickness $2 \varepsilon$, with uniform density $\rho$ and uniform material response described by an elasticity tensor $\mathbb{C}$ endowed with the standard major and minor symmetries ${ }^{10}$. The displacement fields we allow have the form

$$
u(p, \zeta, t)=u_{0}(p, t)+\zeta u_{1}(p, t),
$$

where

$$
u(p, \zeta, t)=f(p, \zeta, t)-(p+\zeta e),
$$

and hence

$$
u_{0}(p, t)=g(p, t)-p, \quad u_{1}(p, t)=l(p, t)-e
$$

(cf. (5.4)). The corresponding linear strains $E(u)=\operatorname{sym}(\operatorname{Grad} u)$ are

$$
E\left(u_{0}, u_{1}\right)=E_{0}\left(u_{0}, u_{1}\right)+\zeta E_{1}\left(u_{1}\right),
$$

with

$$
\begin{aligned}
E_{0}\left(u_{0}, u_{1}\right) & =\operatorname{sym}\left(\operatorname{grad} u_{0}+u_{1} \otimes e\right), \\
E_{1}\left(u_{1}\right) & =\operatorname{sym}\left(\operatorname{grad} u_{1}\right) .
\end{aligned}
$$

Such strains generate stresses

$$
\widehat{S}\left(u_{0}, u_{1}\right)=\widehat{S}_{0}\left(u_{0}, u_{1}\right)+\zeta \widehat{S}_{1}\left(u_{1}\right),
$$

\footnotetext{
${ }^{10}$ The elasticity tensor $\mathbb{C}$ has major symmetry if $A \cdot(\mathbb{C} B)=B \cdot(\mathbb{C} A)$ for all tensors $A, B ;$ the minor symmetries require $\mathbb{C} A=\mathbb{C}(\operatorname{sym} A)=\operatorname{sym}(\mathbb{C} A)$ for each tensor $A$.
} 
through

$$
\begin{aligned}
\widehat{S}_{0}\left(u_{0}, u_{1}\right) & =\mathbb{C} E_{0}\left(u_{0}, u_{1}\right), \\
\widehat{S}_{1}\left(u_{1}\right) & =\mathbb{C} E_{1}\left(u_{1}\right) .
\end{aligned}
$$

Since we are interested in free vibrations, we include only inertial distanceactions. Accordingly, the two-dimensional evolution equations (5.8) take the form:

$$
\begin{aligned}
\operatorname{div} \widehat{N}\left(u_{0}, u_{1}\right) & =2 \varepsilon \rho \ddot{u}_{0}, \\
\operatorname{div} \widehat{M}\left(u_{1}\right)-\widehat{N}\left(u_{0}, u_{1}\right) e & =\frac{2}{3} \varepsilon^{3} \rho \ddot{u}_{1},
\end{aligned}
$$

where, using (4.6), (4.15b), and (7.3), we see that

$$
\begin{gathered}
\widehat{N}\left(u_{0}, u_{1}\right)=2 \varepsilon \widehat{S}_{0}\left(u_{0}, u_{1}\right)=2 \varepsilon \mathbb{C}\left(\operatorname{grad} u_{0}+u_{1} \otimes e\right), \\
\widehat{M}\left(u_{1}\right)=\frac{2}{3} \varepsilon^{3} \widehat{S}_{1}\left(u_{1}\right)=\frac{2}{3} \varepsilon^{3} \mathbb{C}\left(\operatorname{grad} u_{1}\right) .
\end{gathered}
$$

Combination of (7.4) and (7.5) produces the system of (vectorial, second-order) partial differential equations which govern the free vibrations of linearly elastic plates:

$$
\begin{aligned}
\operatorname{div}\left(\mathbb{C}\left(\operatorname{grad} u_{0}+u_{1} \otimes e\right)\right) & =\rho \ddot{u}_{0}, \\
\operatorname{div}\left(\mathbb{C}\left(\operatorname{grad} u_{1}\right)\right)-3 \varepsilon^{-2}\left(\mathbb{C}\left(\operatorname{grad} u_{0}+u_{1} \otimes e\right)\right) e & =\rho \ddot{u}_{1} .
\end{aligned}
$$

\section{Remarks}

- We need not concern ourselves with the symmetry condition on stress discussed in Section 5.2, as the symmetry of the generalized stresses (7.5) is a consequence of the constitutive prescription (7.3) for the three-dimensional stress ( $c f$. footnote 10 on page 25 ).

- We believe it sensible to choose the elasticity tensor $\mathbb{C}$ so as to model a monoclinic material response with respect to the direction $e$ perpendicular to the base surface. Monoclinic response is indeed the most general response that assigns a privileged role to one given material axis ${ }^{11}$. There is no formal reason either to restrict attention to this special material symmetry or to insist that the privileged response direction agrees with the plate geometry. Of course, such assumptions, when combined, induce

\footnotetext{
${ }^{11}$ A monoclinic elasticity tensor $\mathbb{C}$ has thirteen independent components (elastic moduli) at most; the transversely isotropic subcase, to be studied hereafter, has only five, and there are cases of intermediate complication (Lembo and Podio-Guidugli, 1991).
} 
a much simpler theory, and in fact none of the established plate theories departs from this convenient habit.

Renouncing to have a privileged axis - be it because the material response is more general than monoclinic or because the material is monoclinic with respect to an axis different from $e$-allows for coupling effects that may be worth studying, such as those, say, between bending and stretching deformations. This is a fortiori true when plate- or rod-like electroelastic and magnetoelastic actuators and sensors are under study: the variety of piezoelectric or magnetostrictive effects descending from the constitutive coupling inherent to theories more encompassing than mere elasticity would be further increased by ceasing to insist on coherency between the geometry of local material response and the global geometry of the plate or rod.

\subsection{Shearing and Thickness Oscillations}

We now specialize the treatment of the preceding subsection so as to cover oscillations of plates with transversely isotropic response with respect to the direction $e$ perpendicular to the base surface. To represent transversely-isotropic elasticity tensors, from the many alternatives (Lembo and Podio-Guidugli, 1991; Podio-Guidugli, forthcoming) we choose the one used by Podio-Guidugli (forthcoming), namely,

$$
\begin{aligned}
\mathbb{C}= & \gamma_{1}\left(C_{1} \otimes C_{1}+C_{2} \otimes C_{2}\right)+\gamma_{2}\left(C_{3} \otimes C_{3}+C_{4} \otimes C_{4}\right)+ \\
& \delta_{1} D_{1} \otimes D_{1}+\delta_{2}\left(D_{1} \otimes D_{2}+D_{2} \otimes D_{1}\right)+\delta_{3} D_{2} \otimes D_{2} .
\end{aligned}
$$

Here, for $\left\{c_{1}, c_{2}, e\right\}$ an orthonormal basis for $\mathcal{V}$, the set of tensors

$$
\left\{C_{1}, C_{2}, C_{3}, C_{4} ; D_{1}, D_{2}\right\}
$$

defined by

$$
\begin{aligned}
\sqrt{2} C_{k} & =c_{k} \otimes e+e \otimes c_{k}, \quad k=1,2, \\
\sqrt{2} C_{3} & =c_{1} \otimes c_{2}+c_{2} \otimes c_{1}, \\
\sqrt{2} C_{4} & =c_{1} \otimes c_{1}-c_{2} \otimes c_{2}, \\
D_{1} & =e \otimes e \\
\sqrt{2} D_{2} & =c_{1} \otimes c_{1}+c_{2} \otimes c_{2},
\end{aligned}
$$

is an orthonormal basis for the space of symmetric tensors. Moreover, the elastic moduli are assumed to satisfy the positivity requirements

$$
\gamma_{1}>0, \quad \gamma_{2}>0, \quad \delta_{1}>0, \quad \delta_{1} \delta_{3}-\left(\delta_{2}\right)^{2}>0,
$$


a set of inequalities necessary and sufficient for the stored-energy density to be positive. In particular, the isotropic case obtains from (7.7a) when

$$
\gamma_{1}=\gamma_{2}=2 \mu, \quad \delta_{1}=\lambda+2 \mu, \quad \delta_{2}=\sqrt{2} \lambda, \quad \delta_{3}=2(\lambda+\mu),
$$

with $\lambda$ and $\mu$ the Lamé moduli.

To concentrate on the simplest shearing and thickness oscillations, we rule out any other than in-plane motions of the base surface $\mathfrak{P}$ by assuming that

$$
u_{0} \cdot e=0 \text {. }
$$

Under this hypothesis, and with the constitutive prescription (7.7), the two evolution equations (7.6) yield, respectively,

$$
\begin{aligned}
\gamma_{2} \operatorname{div}\left(\operatorname{grad} u_{0}\right)+\delta_{3} \operatorname{grad}\left(\operatorname{div} u_{0}\right)+\sqrt{2} \delta_{2} \operatorname{grad} \tau & =2 \rho \ddot{u}_{0}, \\
\operatorname{div} \tilde{u}_{1} & =0,
\end{aligned}
$$

and

$$
\begin{aligned}
\gamma_{2} \operatorname{div}\left(\operatorname{grad} \tilde{u}_{1}\right)+\delta_{3} \operatorname{grad}\left(\operatorname{div} \tilde{u}_{1}\right)-3 \varepsilon^{-2} \gamma_{1} \tilde{u}_{1} & =2 \rho \ddot{\tilde{u}}_{1}, \\
\gamma_{1} \operatorname{div}(\operatorname{grad} \tau)-3 \varepsilon^{-2}\left(2 \delta_{1} \tau+\sqrt{2} \delta_{2} \operatorname{div} u_{0}\right) & =2 \rho \ddot{\tau},
\end{aligned}
$$

where we have set

$$
\tilde{u}_{1}=P u_{1}, \quad \tau=u_{1} \cdot e,
$$

singling out the fiber elongation $\tau$.

Clearly, the system (7.11) decouples into the subsystem consisting of (7.11a) and (7.11d), which determines the vector field

$$
u_{0}(p, t)+\zeta \tau(p, t) e ;
$$

and the remaining subsystem, which determines the vector field

$$
\zeta \tilde{u}_{1}(p, t) .
$$

The fields (7.13) and (7.14), which (not surprisingly) turn out to be energetically orthogonal, add up to the displacement field $u$.

We conclude that transversely isotropic plates are capable of two types of free vibrations with no transverse motion of their base surface: transverse shearing oscillations (7.14), in which the base surface does not move and the thickness does not change with time (to within the approximation intrinsic to the linear theory); and in-plane/thickness oscillations (7.13), in which the directors, whose length may change with time, remain orthogonal to the base surface, while the latter may deform within its plane.

Notice that (7.11a) and (7.11d) further decouple when $\delta_{2}=0$, a constitutive instance compatible with the positivity requirements (7.8) and describing plates for which thickness changes and base-area changes are uncoupled. 


\subsection{Propagation of Thickness-Distention Waves}

Here we wish to investigate whether equations (7.11a), (7.11d) account for propagation of planar progressive thickness-distention waves, i.e., whether there are solutions of the form

$$
\begin{aligned}
u_{0}(p, t) & =\exp \left(2 \pi i\left(a \cdot\left(p-p_{\mathrm{o}}\right)-V t\right) / L\right) U_{\mathrm{o}}, \\
\tau(p, t) & =\exp \left(2 \pi i\left(a \cdot\left(p-p_{\mathrm{o}}\right)-V t\right) / L\right) T_{\mathrm{o}},
\end{aligned}
$$

with nonnull thickness-distention amplitude $\left(T_{\mathrm{O}} \neq 0\right)$; in $(7.15) p_{\mathrm{o}} \in \mathfrak{P}$, the unit vector $a$ (perpendicular to $e$ ) is the direction and $V \geq 0$ the speed of propagation of a harmonic wave of wavelength $L>0$.

A straightforward computation yields the following result: thickness-distention waves are possible for all transversely isotropic materials of type $(7.7)^{12}$, and propagate with any speed $V$ that solves the dispersion equation

$$
\left(V_{\vdash}^{2}-V^{2}\right)\left(V_{\perp}^{2}-V^{2}\right)=\Delta^{2},
$$

where

$$
\begin{aligned}
V_{\vdash}^{2} & =\frac{\gamma_{2}+\delta_{3}}{2 \rho}, \\
V_{\perp}^{2} & =\frac{\gamma_{1}}{2 \rho}+\frac{6}{\pi^{2}} \frac{\delta_{1}}{2 \rho}\left(\frac{L}{2 \varepsilon}\right)^{2}, \\
\Delta^{2} & =\frac{6}{\pi^{2}}\left(\frac{\delta_{2}}{2 \rho}\right)^{2}\left(\frac{L}{2 \varepsilon}\right)^{2} .
\end{aligned}
$$

Notice that - as expected from a bona fide plate theory - the parameter $L$ enters (7.16) through the scaled wavelength $L /(2 \varepsilon)$; and that the coupling modulus $\delta_{2}$ only appears (squared) on the right side of (7.16). Let

$$
\begin{aligned}
& V_{-}=\min \left\{V_{\vdash}, V_{\perp}\right\}, \\
& V_{+}=\max \left\{V_{\vdash}, V_{\perp}\right\} .
\end{aligned}
$$

While $V_{\perp}$ steadily increases with $L, V_{\vdash}$ is independent of it; if

$$
\gamma_{1}<\gamma_{2}+\delta_{3},
$$

there exists a crossover wavelength $L_{\times}$for which $V_{-}=V_{+}$; otherwise, $V_{-}=V_{\vdash}<$ $V_{\perp}=V_{+}$for all wavelengths.

Equation (7.16) is readily solved in the special case $\delta_{2}=0$ : there is only one type of thickness-distention waves ${ }^{13}$, which propagate with speed $V_{\perp}$ leaving the base

\footnotetext{
${ }^{12}$ On the contrary, in-plane transversal waves $\left(U_{\mathrm{o}} \neq 0, U_{\mathrm{o}} \cdot a=0\right)$ with no thickness change $\left(T_{\mathrm{o}}=0\right)$ are exceptional, being only possible if $\delta_{2}=0$; these waves are nondispersive, and propagate with speed $\sqrt{\gamma_{2} /(2 \rho)}$.

${ }^{13}$ These waves were already considered by DeSimone and Podio-Guidugli (1995).
} 
surface motionless $\left(U_{\mathrm{o}}=0\right)$; and there are in-plane, nondispersive, and longitudinal waves of speed $V_{\vdash}$, without thickness changes $\left(U_{\mathrm{o}} \neq 0, U_{\mathrm{o}} \times a=0 ; T_{\mathrm{o}}=0\right)$.

For a generic material with $\delta_{2} \neq 0$ there are two types of thickness-distention waves; each is both longitudinal and dispersive. If $V_{l}$ and $V_{h}$ denote the speeds of these two waves (with $V_{l} \leq V_{h}$ ), it is not difficult to see that, whatever the wavelength,

$$
V_{l}<V_{-} \leq V_{+}<V_{h}
$$

Moreover, $V_{l} \uparrow V_{-}, V_{h} \downarrow V_{+}$for vanishingly small $L$; at the other extreme, for infinitely large $L, V_{h}$ behaves like $V_{\perp}$, while $V_{l}$ tends to the limit value

$$
V_{\infty}=\sqrt{V_{\vdash}^{2}-\frac{\left(\delta_{2}\right)^{2}}{2 \rho \delta_{1}}}
$$

(which is guaranteed to exist, by (7.8)). The accompanying figure illustrates the dispersion relation (7.16) for a generic material satisfying (7.19); the corresponding figure for materials which do not satisfy (7.19), and thus do not display crossover, is not worth including.

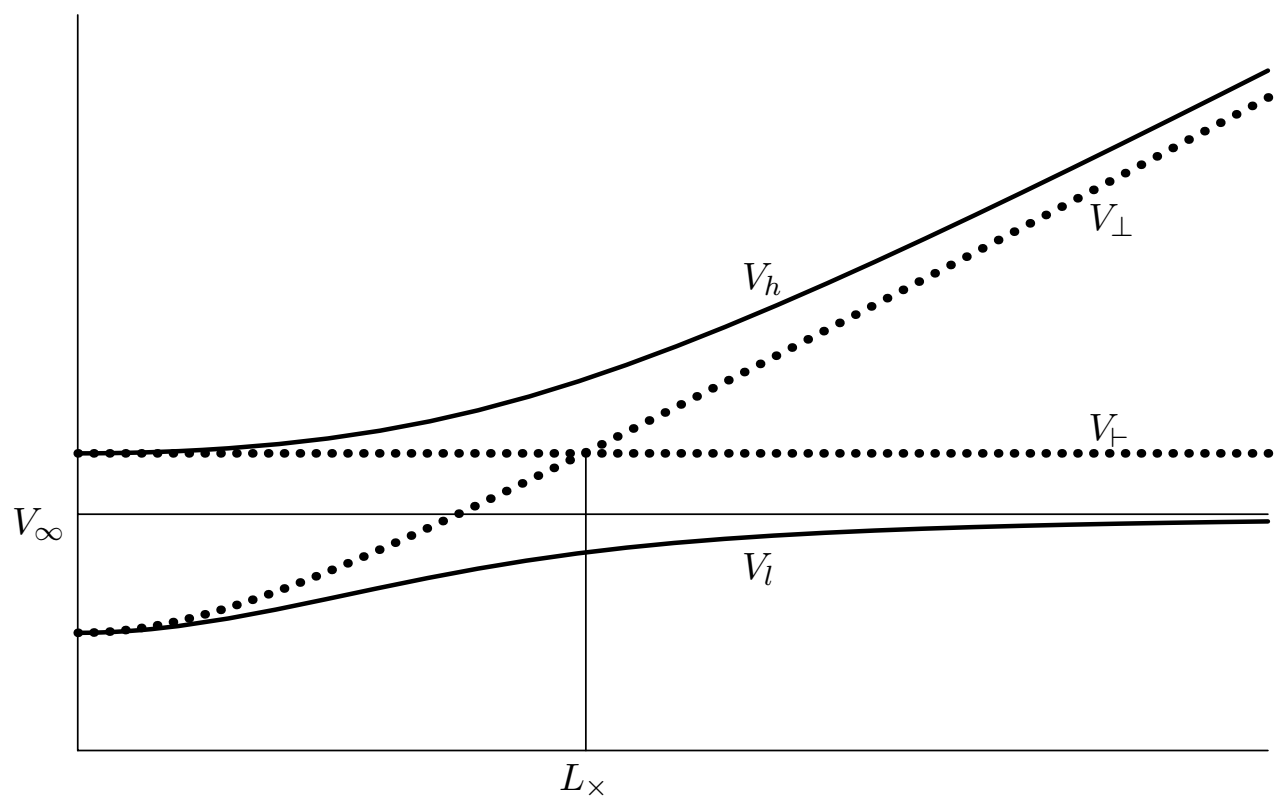

Figure 1: Speed of thickness-distention waves vs. wavelength. 


\subsection{Enriched Kinematics}

In order to demonstrate the effects of kinematical choices that do not force transverse fibers to remain straight and uniformly stretched but rather allow for affine stretch in fibers of uniform curvature, we extend (7.1) by choosing as admissible displacement fields those of the form

$$
u(p, \zeta, t)=u_{0}(p, t)+\zeta u_{1}(p, t)+\zeta^{2} u_{2}(p, t) .
$$

This kinematical assumption amounts to a generalization of the admissible relative motions (3.7) to the form

$$
\mathfrak{g}(p, \zeta, t)=\zeta\left(e+u_{1}(p, t)\right)+\zeta^{2} u_{2}(p, t)
$$

(cf. (7.1)). Such displacements generate the strains

$$
E\left(u_{0}, u_{1}, u_{2}\right)=E_{0}\left(u_{0}, u_{1}\right)+\zeta E_{1}\left(u_{1}, u_{2}\right)+\zeta^{2} E_{2}\left(u_{2}\right)
$$

with $E_{0}\left(u_{0}, u_{1}\right)$ given by $(7.2 \mathrm{~b})$, while

$$
\begin{aligned}
E_{1}\left(u_{1}, u_{2}\right) & =\operatorname{sym}\left(\operatorname{grad} u_{1}+2 u_{2} \otimes e\right), \\
E_{2}\left(u_{2}\right) & =\operatorname{sym}\left(\operatorname{grad} u_{2}\right)
\end{aligned}
$$

and the stresses

$$
\widehat{S}\left(u_{0}, u_{1}, u_{2}\right)=\widehat{S}_{0}\left(u_{0}, u_{1}\right)+\zeta \widehat{S}_{1}\left(u_{1}, u_{2}\right)+\zeta^{2} \widehat{S}_{2}\left(u_{2}\right),
$$

with $\widehat{S}_{0}\left(u_{0}, u_{1}\right)$ given by $(7.3 \mathrm{~b})$, while

$$
\begin{aligned}
\widehat{S}_{1}\left(u_{1}, u_{2}\right) & =\mathbb{C} E_{1}\left(u_{1}, u_{2}\right), \\
\widehat{S}_{2}\left(u_{2}\right) & =\mathbb{C} E_{2}\left(u_{2}\right) .
\end{aligned}
$$

As we have observed at the end of Section 6.2 in connection with our discussion of the expansion scheme presented in Naghdi (1972), when the form of admissible relative motions is so extended as to include terms in $\zeta^{2}$, application of the principle of null working results into both one additional generalized stress, namely, the triple stress

$$
\mathbb{M}(p, t)=\int_{\mathfrak{I}} \zeta^{2} S(p, \zeta, t) d \zeta
$$

and one additional balance equation, where both $\mathbb{M} P$, the base triple stress, and $M e$ enter ${ }^{14}$. Our present plate equations are then three, and have the form:

$$
\operatorname{div}\left(3 \widehat{S}_{0}\left(u_{0}, u_{1}\right)+\varepsilon^{2} \widehat{S}_{2}\left(u_{2}\right)\right)=\rho\left(3 \ddot{u}_{0}+\varepsilon^{2} \ddot{u}_{2}\right),
$$

\footnotetext{
${ }^{14} C f$. the first remark in Section 4.4 ; in turn, $\mathbb{M e}$ would appear within a richer theory, further extended so as to include terms in $\zeta^{3}$.
} 


$$
\begin{aligned}
& \operatorname{div}\left(\varepsilon^{2} \widehat{S}_{1}\left(u_{1}, u_{2}\right)\right)-\left(3 \widehat{S}_{0}\left(u_{0}, u_{1}\right)+\varepsilon^{2} \widehat{S}_{2}\left(u_{2}\right)\right) e=\rho \varepsilon^{2} \ddot{u}_{1} \\
& \operatorname{div}\left(5 \widehat{S}_{0}\left(u_{0}, u_{1}\right)+3 \varepsilon^{2} \widehat{S}_{2}\left(u_{2}\right)\right)-10 \widehat{S}_{1}\left(u_{1}, u_{2}\right) e=\rho\left(5 \ddot{u}_{0}+3 \varepsilon^{2} \ddot{u}_{2}\right) .
\end{aligned}
$$

This system should be compared with the system of two equations (7.4a), (7.4b) ensuing from the $\zeta$-kinematics (7.1), which, in our current notation, can be written as

$$
\begin{aligned}
\operatorname{div} \widehat{S}_{0}\left(u_{0}, u_{1}\right) & =\rho \ddot{u}_{0}, \\
\operatorname{div}\left(\varepsilon^{2} \widehat{S}_{1}\left(u_{1}, 0\right)\right)-3 \widehat{S}_{0}\left(u_{0}, u_{1}\right) e & =\rho \varepsilon^{2} \ddot{u}_{1} ;
\end{aligned}
$$

and with the single equation that, for the $\zeta^{0}$-kinematics (4.25), describes the evolution of the base surface:

$$
\operatorname{div} \widehat{S}_{0}\left(u_{0}, 0\right)=\rho \ddot{u}_{0} .
$$

Examples of solutions of the same three-dimensional sample problem within the $\zeta^{0}$, $\zeta^{1}$, and $\zeta^{2}$-kinematics are given in DiCarlo et al. (forthcoming).

\section{References}

Antman, S.S., Osborne, J.E., 1979. The principle of virtual work and integral laws of motion. Archive for Rational Mechanics and Analysis 69, 231-262.

Antman, S.S., Marlow, R.S., 1991. Material constraints, Lagrange multipliers, and compatibility. Applications to rod and shell theories. Archive for Rational Mechanics and Analysis 116, 257-299.

Antman, S.S., 1995. Nonlinear Problems of Elasticity. Springer-Verlag, New York, pp. $435-436$.

Capriz, G., 1989. Continua with Microstructure. Springer-Verlag, New York.

Cauchy, A.-L., 1827. De la pression ou tension dans un corps solide. In: Exercices de Mathématique, Vol. 2, pp. 42-56. English translation of the quoted excerpt from: Truesdell, C., 1991. A First Course in Rational Continuum Mechanics, Vol. 1 (2nd ed.). Academic Press, Boston, p. 150.

DeSimone, A., Podio-Guidugli, P., 1995. Centri di forza e oscillazioni di spessore nelle piastre. In: Atti del XII Congresso Nazionale di Meccanica Teorica e Applicata, Vol. V. AIMETA, Napoli, pp. 99-104. 
DiCarlo, A., 1996. A non-standard format for continuum mechanics. In: Contemporary Research in the Mechanics and Mathematics of Materials (Batra, R.C., Beatty, M.F., eds.). CIMNE, Barcelona, pp. 263-268.

DiCarlo, A., forthcoming. A clean direct theory of shells with distensible thickness.

DiCarlo, A., Podio-Guidugli, P., Williams, W.O., forthcoming. Approximations within a hierarchical plate theory of a three-dimensional benchmark solution.

Ericksen, J.L., Truesdell, C., 1958. Exact theory of stress and strain in rods and shells. Archive for Rational Mechanics and Analysis 1, 295-323.

Germain, P., 1972. Sur l'application de la méthode des puissances virtuelles en mécanique des milieux continus. Comptes Rendus de l'Académie des Sciences de Paris 274 (Série A), 1051-1055.

Germain, P., 1973a. La méthode des puissances virtuelles en mécanique des milieux continus. Première partie: Théorie du second gradient. Journal de Mécanique $12,235-274$.

Germain, P., 1973b. The method of virtual power in continuum mechanics. Part 2: Microstructure. SIAM Journal on Applied Mathematics 25, 556-575.

Green, A.E., Rivlin, R.S., 1964. Simple force and stress multipoles. Archive for Rational Mechanics and Analysis 16, 325-353.

Green, A.E., Naghdi, P.M., Wainwright, W.L., 1965. A general theory of a Cosserat surface. Archive for Rational Mechanics and Analysis 20, 287-308.

Green, A.E., Laws, N., Naghdi, P.M., 1968. Rods, plates and shells. Proceedings of the Cambridge Philosophical Society 64, 895-913.

Green, A.E., Naghdi, P.M., 1970, 1971. Non-isothermal theory of rods, plates and shells. International Journal of Solids and Structures 6, 209-244; 7, 127-127.

Gurtin, M.E., 1972. The linear theory of elasticity. In: Encyclopedia of Physics, Vol. VIa/2 (Truesdell, C., ed.). Springer-Verlag, Berlin, pp. 1-295.

Lembo, M., Podio-Guidugli, P., 1991. Plate theory as an exact consequence of threedimensional linear elasticity. European Journal of Mechanics A/Solids 10, 485516.

Levinson, M., 1985. The simply supported rectangular plate: an exact, three dimensional, linear elasticity solution. Journal of Elasticity 15, 283-291. 
Maugin, G.A., 1980. The method of virtual power in continuum mechanics: applications to coupled fields. Acta Mechanica 35, 1-70.

Naghdi, P.M., 1972. The theory of shells and plates. In: Encyclopedia of Physics, Vol. VIa/2 (Truesdell, C., ed.). Springer-Verlag, Berlin, pp. 425-640.

Naghdi, P.M., 1981. Finite deformation of elastic rods and shells. In: Proceedings of the IUTAM Symposium on Finite Elasticity (Carlson, D.E., Shield, R.T., eds.). Martinus Nijhoff Publishers, The Hague, pp. 47-103.

Nardinocchi, P., Podio-Guidugli, P., 1994. The equations of Reissner-Mindlin plates obtained by the method of internal constraints. Meccanica 29, 143-157.

Noll, W., 1963. La mécanique classique, basée sur un axiome de objectivité. In: La Méthode Axiomatique dans les Mécaniques Classiques et Nouvelles (Colloque International, Paris, 1959), Gauthier-Villars, Paris, pp. 47-56. Reprinted in: Noll, W.,1974. The Foundations of Mechanics and Thermodynamics. SpringerVerlag, Berlin, pp. 135-144.

Pan, E., 1991. An exact solution for transversely isotropic, simply supported and layered rectangular plates. Journal of Elasticity 25, 101-116.

Podio-Guidugli, P., 1989. An exact derivation of the thin plate equation. Journal of Elasticity 22, 121-133.

Podio-Guidugli, P., 1995. Local and global constraints. Paper presented at the Third Meeting on Current Ideas in Mechanics and Related Fields, Segovia.

Podio-Guidugli, P., forthcoming. Theory of Elastic Plates.

Rogers, T.G., Watson, P., Spencer, A.J.M., 1992. An exact three-dimensional solution for normal loading of inhomogeneous and laminated anisotropic plates of moderate thickness. Proceedings of the Royal Society of London A 437, 199213.

Teresi, L., Tiero, A., 1997. On variational approaches to plate models. Meccanica $32,143-156$.

Truesdell, C., Toupin, R.A., 1960. The classical field theories. In: Encyclopedia of Physics, Vol. III/1 (Flügge, S., ed.). Springer-Verlag, Berlin, pp. 226-793. 